\title{
Young girl diagnosed with chronic recurrent multi-focal osteomyelitis
}

\begin{abstract}
Chronic Recurrent Multi-focal Osteomyelitis is a rare form of inflammatory disorder diagnosed after exclusion of malignancy, and infection. It is rare due to obscure knowledge of physicians regarding this disease and lack of any specific clinical parameter. We present a case of an 8 years old girl who was being managed by infectious disease department for almost a year before being referred to us. She had taken prolonged courses of antimicrobials during this time.
\end{abstract}

Keywords: chronic recurrent multi-focal osteomyelitis • osteomyelitis

\section{Introduction}

Chronic Recurrent Multi-focal Osteomyelitis (CRMO) is a rare entity, and has seldom been described in literature. Lack of awareness and being closely related to bacterial osteomyelitis both symptom and radiological wise makes it a difficult disease to diagnose. Often patients are exposed to prolonged courses of antibiotics and undergo a barrage of tests. Case series reviewed from developed countries, show that average time to diagnose a patient with CRMO has been $1 \frac{1}{2}$ years. As it is an inflammatory condition primarily seen in young children, patients often go through back in forth between infectious disease and orthopedic consultations before finally being diagnosed and referred to rheumatology services.

\section{Case Report}

8 years old girl presented with complaint of gradual onset of pain and swelling in her left leg for over 9 months, which had progressively increased. She had a history of fall prior to onset of symptoms for which she went to an orthopedic surgeon who diagnosed her as having a fracture and applied back slab. Her symptoms persisted and she also developed high grade fever, on \& off, documented at 102-103. She consulted with infectious disease and after imaging she was diagnosed and treated as osteomyelitis. She was started on injection piperacillin+tazobactam for 6 weeks to which she responded, and got better. After antibiotic course her symptoms relapsed and she again consulted infectious disease, who after imaging started her on injection linezolid with NSAIDs, which was given for $31 / 2$ months. Her symptoms again regressed but recurred after $1 \frac{1}{2}$ months of stopping antibiotics. She was then referred to rheumatology clinics. She was able to walk, go to school, but was having difficulty in placing weight on her affected leg. She had no history of TB, TB contact, weight loss, etc.

On examination she was a young girl of average height and built, sitting comfortably on chair and vitally stable. Mild swelling, tenderness over her left lower limb Figure 1, distal $1 / 3^{\text {rd }}$ of leg is warm with erythema present. Rest of her musculoskeletal and systemic examination was unremarkable.

\section{Investigations}

Hb: $12.6 \mathrm{~g} / \mathrm{dl}$, TLC: $7.8 \times 10^{9} / \mathrm{L}$, PLT: 391,000/L, ESR: $56 \mathrm{~mm} / \mathrm{hr}$, SGPT: $51 \mathrm{IU} / \mathrm{L}$, S. Creat: $0.74 \mathrm{mg} / \mathrm{dl}$. Her serially repeated CRP over 9 months were all raised, last one being=21.8 $(<0.5)$, ANA negative, urine DR $\& \mathrm{c} / \mathrm{s}=$ normal, blood $\mathrm{c} / \mathrm{s}=$ showed no growth, complement levels, and immunoglobulin levels were normal, HIV serology negative, CXR (PA view) and echocardiography were normal.

X-Ray left leg showed periosteal reaction and lytic lesions. Initial MRI left leg showed patchy irregular areas of abnormal signal intensity involving whole length of tibia with increase signals, thickening and edema in adjacent soft tissues and subcutaneous fat. Findings were said to be suggestive of osteomyelitis.
Tahira Perveen Umer, Lubna Nazir \& Hamza Alam* Department of Rheumatology, Liaquat National Hospital, Karachi, Pakistan

*Author for correspondence: drhamza84@gmail.com 
A repeat MR after 3 months of antibiotics showed similar findings, with slight interval change. Another repeat MR after second course of antibiotic showed re-demonstration of abnormal signals and enhancement of entire tibia with cloaca formation and adjacent soft tissue thickening/enhancement which showed progression. Overall findings were suggestive of chronic osteomyelitis Figure 2.

A three phase skeletal scintigraphy was advised to rule out involvement of other bones. The findings are consistent with chronic osteomyelitis involving whole shaft of left tibia only with evidence of cellulitis over distal $1 / 3^{\text {rd }}$ of leg Figure 3.

Bone biopsy showed predominantly fibro collagenous tissue with fragments of sclerotic bone. Bone c/s, fungal smear and c/s showed no growth, AFB smear and c/s were also negative.

\section{Further Management}

Due to repeated negative blood cultures and unable to yield an organism on bone cultures we diagnosed patient as having chronic recurrent multi-focal osteomyelitis. She was given injection pamidronate $1.5 \mathrm{mg} / \mathrm{kg}$ to which she responded and improved, her CRP reduced and repeat $\mathrm{x}$-ray showed remarkable improvement. Plan is to repeat MR to screen for disease activity.

\section{Discussion}

Chronic recurrent multifocal osteomyelitis (CRMO), is an inflammatory bone disease. It occurs primarily in children and adolescents and is unfamiliar to many. It was first described in 1972 by Giedion as "an unusual form of multifocal bone lesions with sub-acute and chronic symmetrical osteomyelitis" [1]. There is a paucity of CRMO cases described in the

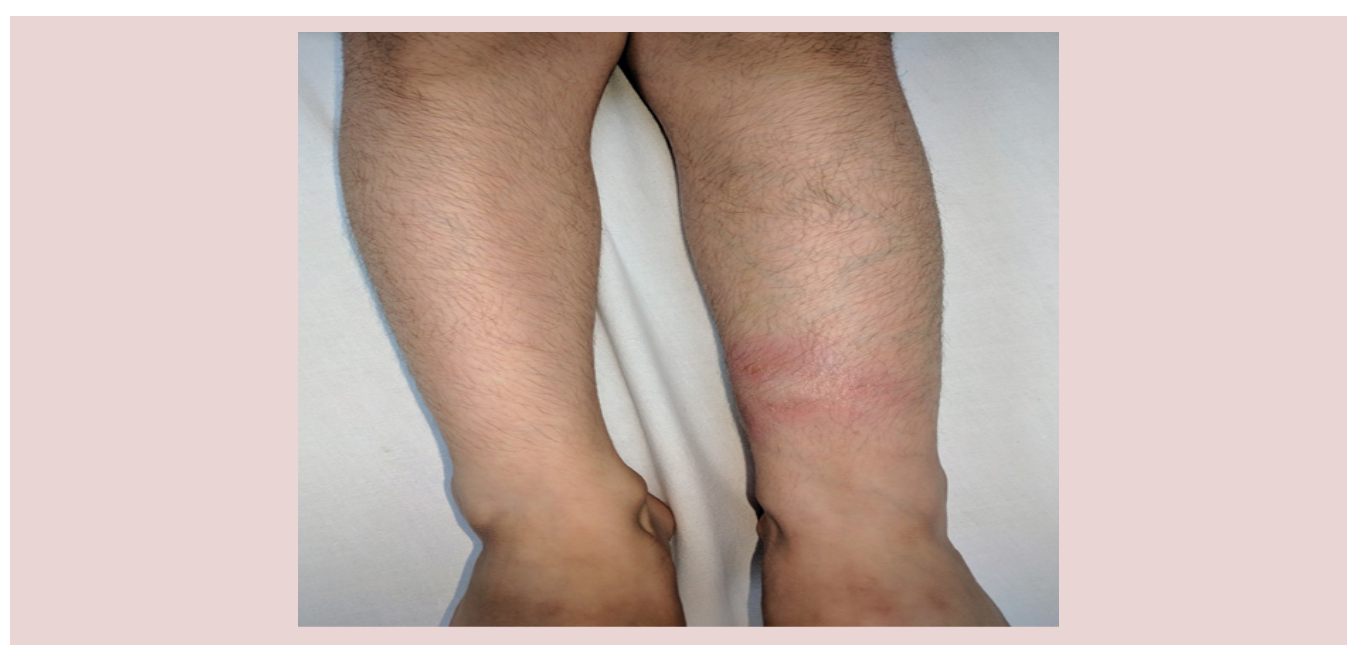

Figure 1. Evident swelling and redness over left leg

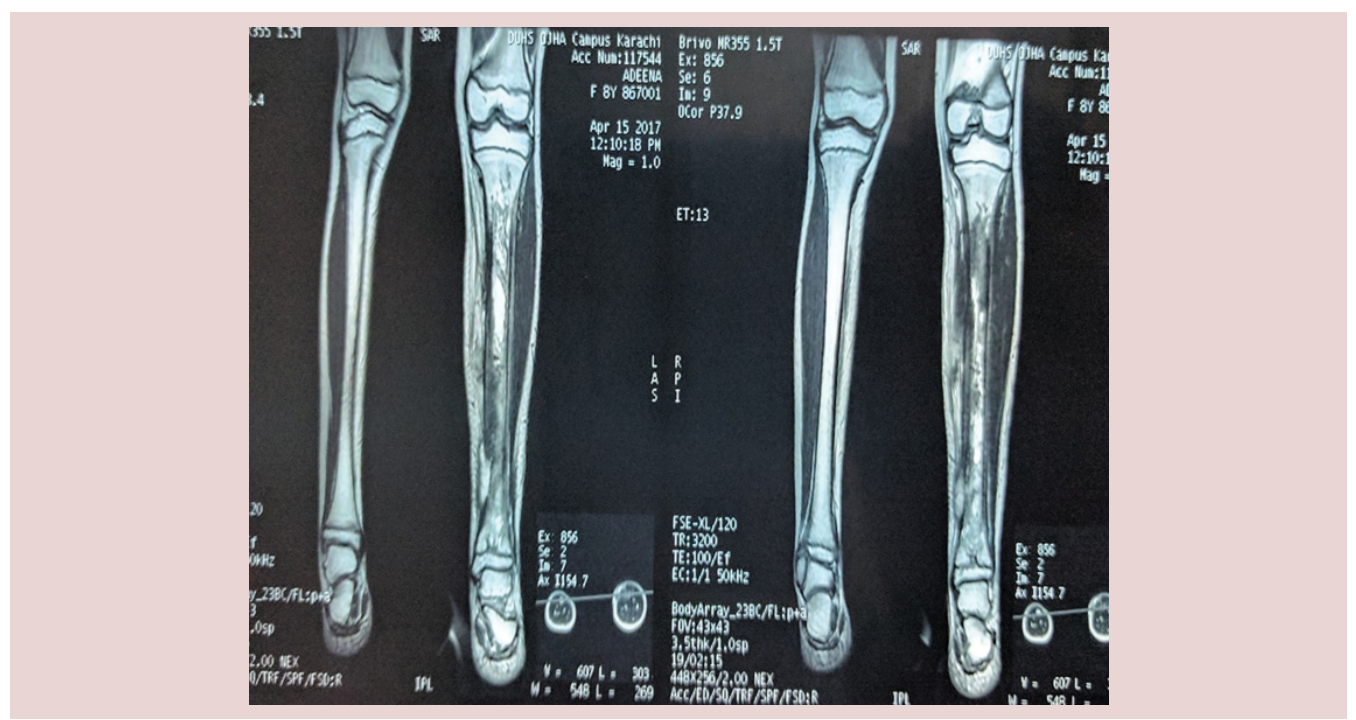

Figure 2. MRI showing patchy irregular areas with abnormal intensity signals 


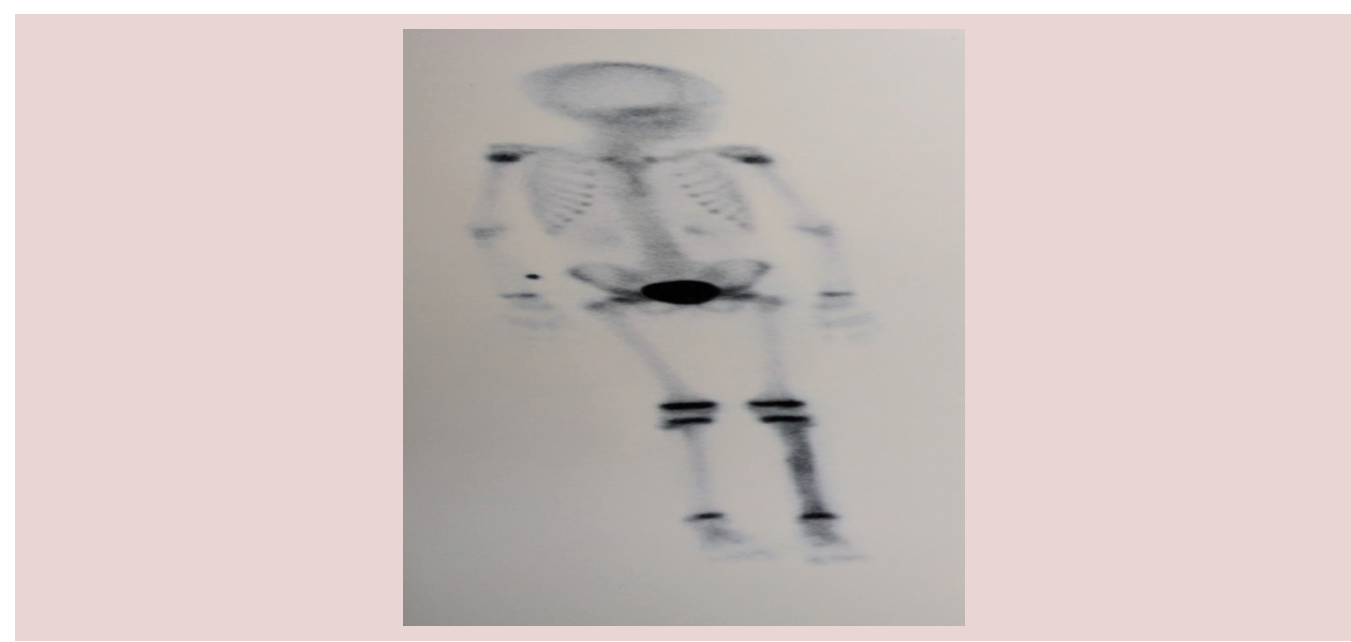

Figure 3. Bone scan showing chronic osteomyelitis over left tibia

literature. They are predominantly case series. True prevalence of CRMO is difficult to assess and likely to be vastly underdiagnosed [2].

The consistent feature of CRMO is the insidious onset of pain, swelling and tenderness which is localized over the affected bones. Involvement of the clavicle is the classical picture; however, femur, tibia or humerus are also frequently affected. Lesions can affect any bone [3]. As the condition is obscure to many doctors, and commonly being misdiagnosed as bacterial osteomyelitis, delays in referral and correct diagnosis are seen. This may lead to prolonged antibiotic courses (often given intravenously in hospital), unnecessary radiation exposure from multiple plain radiographs or bone scans and repeated bone biopsies before a diagnosis is made.

The diagnosis of CRMO is made by exclusion of other diseases especially infection and neoplasia, hence commonly requires a bone biopsy [4]. Many doctors and articles described CRMO as an autoimmune disease that has symptoms and radiological features similar to osteomyelitis, but without the infection.

The empirical diagnostic criteria for CRMO include the following: multifocal bone lesions, a clinical course extended for months with remissions and exacerbations, a lack of response to antimicrobial treatment, osteolytic-sclerotic bone lesions on X-ray. Compliance with all of the criteria is required for a definitive diagnosis.

Similarly our patient a young girl, who had signs, symptoms and radiographic features consistent with chronic osteomyelitis. Despite her extensive workup and treatment she continued to remain symptomatic. Numerous cultures, bone biopsy failed to yield any definitive organism. It is also worth mentioning that she improved clinically for a brief period on her $1^{\text {st }}$ course of antibiotic. It is well documented that there are a few antibiotics are known to have an anti-inflammatory effect; tazobactam might also have similar effects.

Different treatment options have been postulated. Studies show that azithromycin showed fast clinical improvement in patients. Its therapeutic effect in patients with CRMO was surprising and lead to the hypothesis that azithromycin could have additional anti-inflammatory effect [5] NSAIDS are the treatment of choice for CRMO. Other treatment options defined are corticosteroids, pamidronate, sulfasalazine, methotrexate, Gamma INF and INF alfa blockage, which have been reported with some success.

Our patient improved clinically on injection pamidronate, and her CRP improved. We plan to follow her up with repeat MR and look for resolution of radiographic changes.

\section{References}

1. Giedion A, Holthusen W, Masel LF et al. Subacute and chronic "symmetrical" osteomyelitis. Ann. Radiol. 15, 329-342 (1972).

2. Iyer RS, Thapa MM, Chew FS. Chronic recurrent multifocal osteomyelitis: review. Am. J. Roentgenol. 196, S87-S91 (2011).

3. Catalano-Pons C, Comte A, Wipff J et al. Clinical outcome in children with chronic recurrent multifocal osteomyelitis. Rheumatology (Oxford). 47, 1397-1399 (2008).

4. Roderick MR, Shah R, Rogers V et al. Chronic recurrent multifocal osteomyelitis (CRMO) advancing the diagnosis. Pediatr. Rheumatol. Online. J. 14(1), 47 (2016).

5. Schilling F, Wagner AD. Azithromycin: an antiinflammatory effect in chronic recurrent multifocal osteomyelitis? A preliminary report. Z. Rheumatol. 59(5), 352-3 (2000). 